\title{
An overview of head tracking integral imaging three-dimensional display using smart pseudoscopic-to-orthoscopic conversion
}

\author{
Xin Shen ${ }^{1}$, Manuel Martinez-Corral ${ }^{2}$, and Bahram Javidi ${ }^{1 *}$ \\ ${ }^{1}$ Electrical and Computer Engineering Department, University of Connecticut, Storrs, CT 06269- \\ 4157, USA; \\ ${ }^{2}$ Department of Optics, University of Valencia, E-46100 Burjassot, Spain.
}

\begin{abstract}
We overview a previously reported head tracking integral imaging three-dimensional (3D) display to extend viewing angle accommodated to a viewer's position without the crosstalk phenomenon. A head detection system is applied to obtain the head position and rotation of a viewer, and a new set of elemental images is then computed using the smart pseudoscopic-to-orthoscopic conversion (SPOC) method for head tracking 3D display. Experimental results validate the proposed method for high quality 3D display with large viewing angle.
\end{abstract}

Keywords: Integral imaging, pseudoscopic-to-orthoscopic conversion, 3D image processing; 3D display, head tracking.

\section{INTRODUCTION}

Integral imaging (InIm) [1]-[3] is an autosteroscopic three-dimensional (3D) imaging technology which can provide continuous viewing points and 3D visualization without any special viewing glasses. A 3D scene can be recorded as multi-perspective 2D images by using a single imaging sensor with a lenslet array or an array of imaging sensors [4]. The 2D images capture both the directional information and intensity information of a scene. Integral imaging 3D optical reconstruction is the reverse process of the $3 \mathrm{D}$ sensing. The multi-perspective $2 \mathrm{D}$ images are displayed on a display screen, light rays from the screen pass through a lenslet array and integrate in the space to form a 3D image [5].

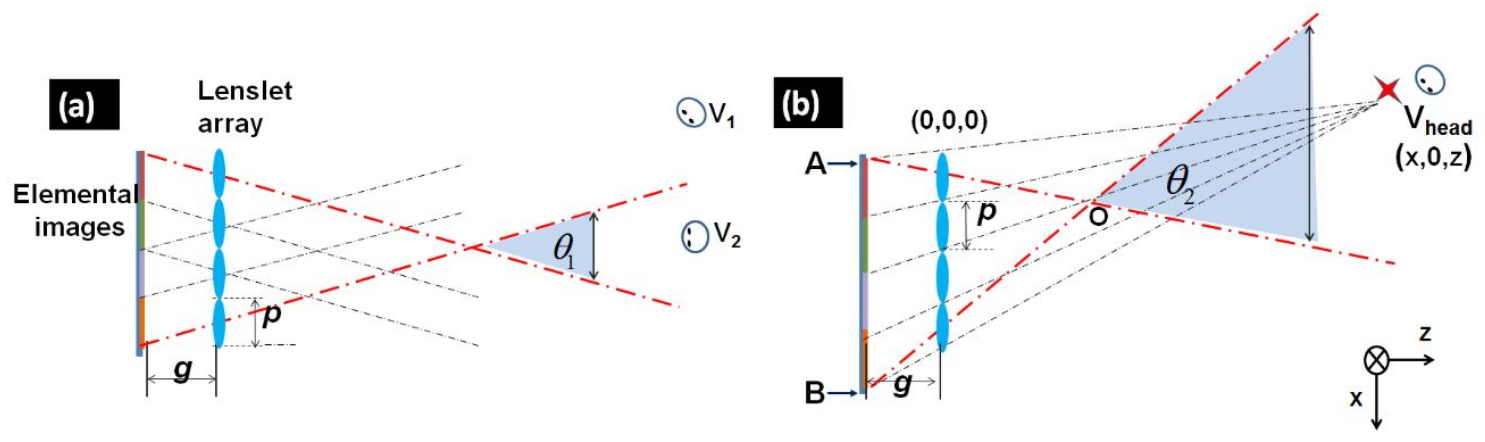

Fig. 1. The viewing angle of an InIm display. (a) For the conventional InIm display. (b) For the head tracking InIm with a specific viewing position. $p$ is the pitch of the lenslet, $g$ is the distance between the display panel and the lenslet, $\theta_{I}$ and $\theta_{2}$ are the viewing angles of conventional and head tracking InIm, respectively [14].

The main performance factors for a conventional integral imaging display system include the resolution quality, 3D image depth of focus, and the viewing angle. Methods and techniques are introduced to improve the performance of the integral imaging 3D display system [5]-[8]. For a conventional InIm optical display, the viewing angle depends on (1) the distance between the elemental image and the lenslset, and (2) the pitch of each lenslet. As shown in Fig. 1(a), high quality $3 \mathrm{D}$ image is provided within the angle $\theta_{1}=2 \arctan (p / 2 g)$ [9]. If observed with an angle larger than $\theta_{l}$, pixels in one elemental image may pass through the adjacent lenslet to form a $3 \mathrm{D}$ image, which may cause the crosstalk 
phenomenon and degrades the 3D image quality. Research works have been done to analyze and solve the problem [10][13].

In this paper, we present an overview of a previously reported head tracking InIm 3D display system [14] to enhance the viewing angle using the smart pseudoscopic-to-orthoscpic conversion (SPOC) method [15][16]. Head tracking technology is considered for 3D display. A head tracking system is added for obtaining the observer's viewing parameters. The elemental images will be re-generated corresponding to the detected viewing positon. Fig. 1(b) illustrates the viewing angle of the head tracking InIm 3D display using new elemental images generated with the observer's positon. This paper is organized as follow: Section. 2 describes the process about the generation of a new elemental image array using the SPOC method. Then the experimental results are explained in Section. 3, and the conclusion is given in Section. 4.

\section{GENERATION OF AN ELEMENTAL IMAGE ARRAY FOR HEAD TRACKING 3D DISPLAY}

The SPOC method is first introduced in [15] to solve the depth reverse problem of the InIm optical display with full control of the system parameters [17]. The previously proposed method for head tracking 3D display using SPOC is depicted in Fig. 2. By using the SPOC method, a new set of elemental images is computationally generated combing the real captured elemental images and the detected observer's viewing position with fully controllable system parameters.

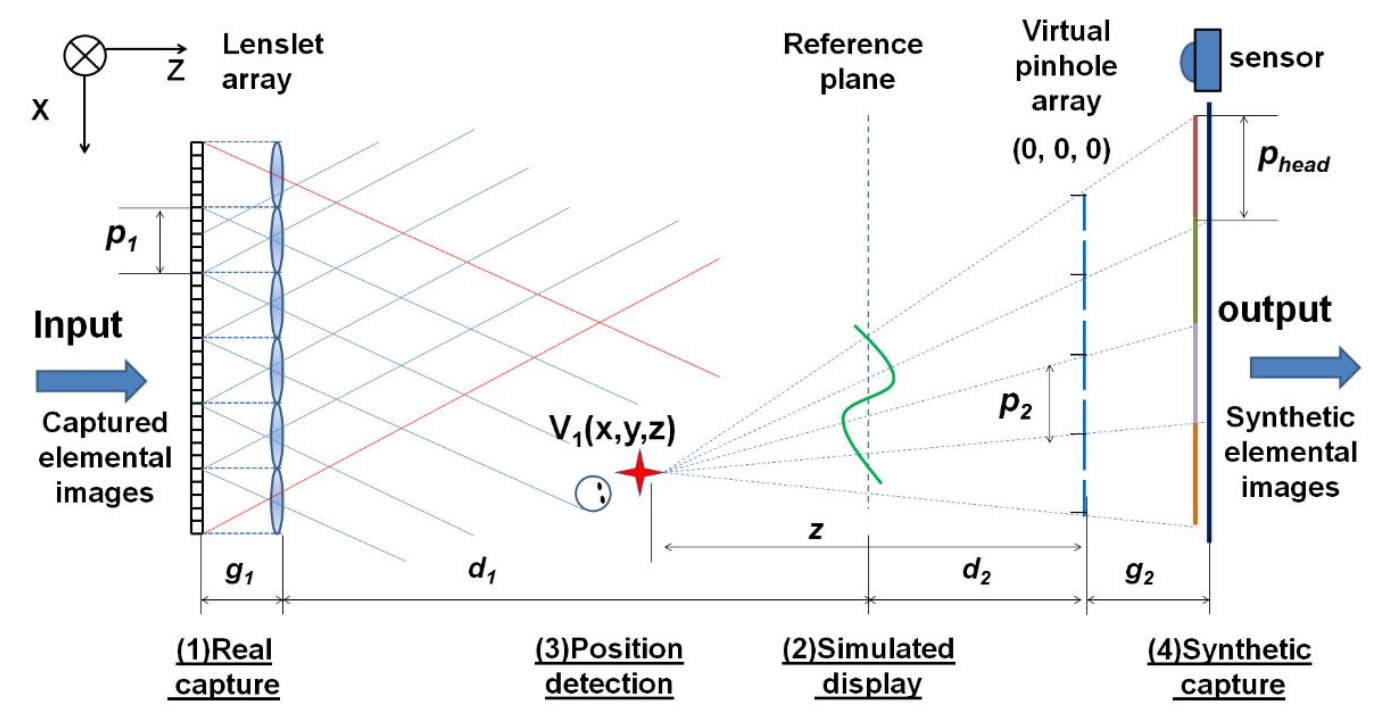

Fig. 2. Generation of a new set of elemental images for the previously reported head tracking InIm 3D display. In the pickup stage, $p_{I}$ is the pitch of the lenslet, $g_{l}$ is the distance between the display screen and the lenslet, and $d_{l}$ is the distance from the lenslet to the reconstruction plane. In the synthetic capture stage, all the system parameters depend on the display system. $d_{2}$ is the distance between the reference plane and the virtual pinhole array, $g_{2}$ is the distance between the virtual pinholes and the synthetic elemental images, and $p_{2}$ is the pitch of the virtual pinholes [14].

There are four general steps for the previously proposed head tracking InIm 3D display method [14]: (1) Pickup process of InIm. A 3D scene is captured based on the conventional InIm, the 3D information is recorded as a set of elemental images with different perspectives, which is named the capture elemental images. (2) Simulated display. The captured elemental images are computationally reconstructed in the 3D space with various in-focus depth planes. (3) Viewing position detection. A head tracking system is used to detect and calculate the observer's viewing position. (4) Synthetic capture. A virtual pinhole array model is set based on the 3D display system and the detected viewing position to generate a new set of synthetic elemental images for head tracking 3D display.

An IR sensor is placed on the plane which is identical with the display system, the signal from an IR clip which is attached with the observer is captured by the sensor and then calculate the viewing position. Once the viewing position is obtained, pixel mapping from the captured elemental images to the synthetic elemental images is conducted through the detected viewing position and the pinhole array model. Note that the parameters in the simulated display stage are based on the real pickup process, however, the parameters in the synthetic capture stage depends on the 3D display system. As 
an advantage of the SPOC method, the synthetically generated elemental images are fully controllable with all the parameters [15][17], it works well even if the corresponding parameters in the pickup process are different with the 3D display system.

\section{EXPERIMENTAL RESULTS}

We show the experimental results between the previously proposed head tracking 3D InIm display system [14] and a conventional 3D display. A head tracking device (TrackIR 5.2TM) was used in the experiment. The sensor was located on the display plane to obtain the observer's viewing position. A 3D scene (letters "3" and "D") was generated by a software (3dsMax). The letters "3" and "D" are located at $40 \mathrm{~mm}$ and $60 \mathrm{~mm}$ from the sensor, respectively. With the captured elemental images, a reference plane was set in the center of the 3D scene $\left(d_{l}=50 \mathrm{~mm}\right)$ for simulated display. The resolution of the display screen (HTC-One) is $1920(\mathrm{H}) \times 1080(\mathrm{~V})$ pixels with the pixel size of $\sim 54 \mu \mathrm{m}$. The pitch of the lenslet is $1 \mathrm{~mm}$, and the distance $\left(g_{2}\right)$ between the lenslet array and the display screen is $3.3 \mathrm{~mm}$. We set the distance $\left(d_{2}\right)$ between the reference plane and the virtual pinhole array as $20 \mathrm{~mm}$. As a result, the 3D image can be displayed around $20 \mathrm{~mm}$ from the display screen. The specifications of the real capture and synthetic capture are shown in Table I [14].

Table I. Specifications of the real capture and synthetic capture for the experiments [14].

\begin{tabular}{lll}
\hline \hline Specifications & Real capture & Synthetic capture \\
\hline$g(\mathrm{~mm})$ & $6.03\left(g_{l}\right)$ & $3.30\left(g_{2}\right)$ \\
\hline$d(\mathrm{~mm})$ & $50\left(d_{1}\right)$ & $20\left(d_{2}\right)$ \\
\hline Elemental images $(\mathrm{EI})$ & $7(\mathrm{H}) \times 7(\mathrm{~V})$ & $104(\mathrm{H}) \times 58(\mathrm{~V})$ \\
\hline Size of each lenslet $(\mathrm{mm})$ & $5(\mathrm{H}) \times 5(\mathrm{~V})$ & $1(\mathrm{H}) \times 1(\mathrm{~V})$ \\
\hline Resolution of EI (pixels) & $2000(\mathrm{H}) \times 2000(\mathrm{~V})$ & $18(\mathrm{H}) \times 18(\mathrm{~V})$ \\
\hline \hline
\end{tabular}

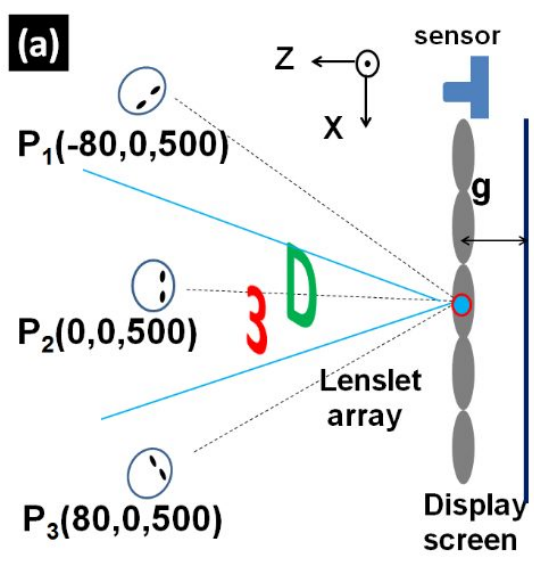

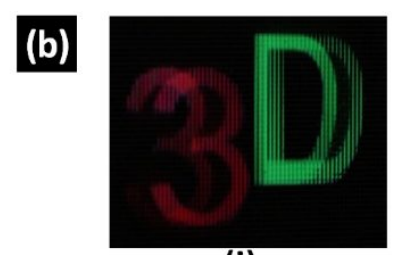

(i)
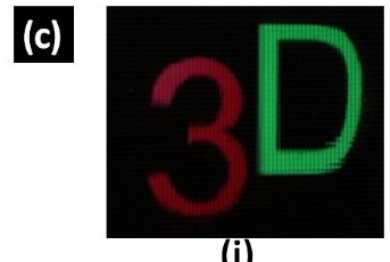

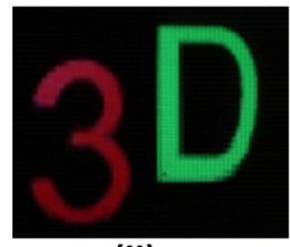

(i) (ii)

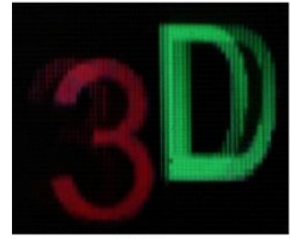

(iii)

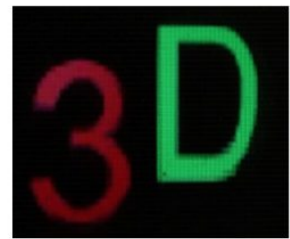

(ii)

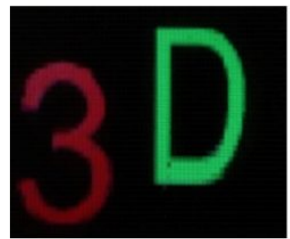

(iii)

Fig. 3. (a) $3 \mathrm{D}$ display with different viewing positions (left view $\mathrm{P}_{1}$, center view $\mathrm{P}_{2}$, and right view $\mathrm{P}_{3}$ ). Display results with (b) conventional InIm, and (c) head tracking InIm. Display results from (i) left view, (ii) center view and (iii) right view [14].

In the experiment, the acceptable viewing angle (FOV) of the conventional InIm is $17.2^{\circ}\left( \pm 8.6^{\circ}\right)$. If the viewing position is out of the FOV, the display results will be degraded due to the crosstalk. Fig. 3(a) illustrates the configuration of the head tracking 3D display experiment. Three positions $\left(\mathrm{P}_{\mathrm{i}}, \mathrm{i}=1,2,3\right)$ were selected for observation. Fig. 3(b) shows the 
corresponding display results by the conventional InIm. The viewing positions at $\mathrm{P}_{1}$ and $\mathrm{P}_{3}$ are out of the FOV. The observed 3D images are flipped. With the previously proposed head tracking 3D display, the display results are improved without image flipping at $\mathrm{P}_{1}$ and $\mathrm{P}_{3}$, as shown in Fig. 3(c). If the 3D image is observed from the center position $\left(\mathrm{P}_{2}\right)$, both the conventional and head tracking $3 \mathrm{D}$ display can provide high quality display results, as shown in Figs. 3(b)(ii) and (c)(ii), respectively.

\section{CONCLUSIONS}

We present an overview of a previously reported head tracking integral imaging (InIm) 3D display for an extended viewing angle [14]. By implementing the smart pseudoscopic-to-orthoscopic conversion (SPOC) method, a new set of elemental images corresponding to a specific viewing position is computationally generated for head tracking 3D display. The experimental results show that the proposed system can eliminate crosstalk for a large viewing angle 3D display.

\section{REFERENCES}

[1] Lippmann, G., "La photographie integrale," CR Acad.Sci 146, 446-451 (1908).

[2] Ives, H. E., "Optical properties of a Lippman lenticulated sheet," J. Opt. Soc. Am. A 21, 171 (1931).

[3] Okano, F., Hoshino, H., Arai, J., and Yuyama, I., "Real-time pickup method for a three-dimensional image based on integral photography," Appl. Opt., 36(7), 1598-1603 (1997).

[4] Jang, J., and Javidi, B., "Three-dimensional synthetic aperture integral imaging," Opt. Lett. 27(13), 1144-1146 (2002).

[5] Martinez-Cuenca, R., Saavedra, G., Martinez-Corral, M. and Javidi, B., "Progress in 3-D multiperspective display by integral imaging," Proc. IEEE 97, 1067-1077 (2009).

[6] Cho, M. J., Daneshpanah, M., Moon, I., and Javidi, B., "Three-Dimensional Optical Sensing and Visualization Using Integral Imaging, " Proc. IEEE, 99(4), 556-575 (2011).

[7] Xiao, X., Javidi, B., Martinez-Corral, M. and Stern, A., "Advances in three-dimensional integral imaging: sensing, display, and applications, " Appl. Opt. 52, 546-560 (2013).

[8] Javidi, B., Shen, X., Markman, A. S., Latorre-Carmona, P., Martínez-Us, A., Sotoca, J. M., Pla, F., Martínez-Corral, M., Saavedra, G., Huang, Y. P., and Stern, A., "Multidimensional Optical Sensing and Imaging Systems (MOSIS): From Macro to Micro Scales," Proc. IEEE, vol. pp, no.99, 1-25 (2017) doi: 10.1109/JPROC.2017.2654318.

[9] Park, J. H., Shin, M.Y., and Kim, N., "Viewing Direction Controllable Three-Dimensional Display Based on Integral Imaging," SID Symposium Digest of Technical Papers, 40(1), 607-610, (2009).

[10] Chen, C. W., Cho, M. J., Huang, Y. P., and Javidi, B., "Improved Viewing Zones for Projection Type Integral Imaging 3D Display Using Adaptive Liquid Crystal Prism Array," J. Display Technol., 10(3), 198-203 (2014).

[11] Martínez-Cuenca, R., Navarro, H., Saavedra, G., Javidi, B., and Martinez-Corral, M., "Enhanced viewing-angle integral imaging by multiple-axis telecentric relay system, " Opt. Express 15(24), 6255-16260 (2007).

[12]Park, G., Hong, J., Kim, Y., and Lee, B., "Enhancement of Viewing Angle and Viewing Distance in Integral Imaging by Head Tracking, " OSA Technical Digest, DWB27 (2009).

[13] Jen, TH, Shen, X, Yao, G, Huang, YP, Shieh, HP, and Javidi, B., "Dynamic integral imaging display with electrically moving array lenslet technique using liquid crystal lens," Opt. Express. 23(14), 18415-18421 (2015).

[14] Shen, X., Martinez-Corral, M., and Javidi, B., "Head tracking three-dimensional integral imaging display using smart pseudoscopic-to-orthoscopic conversion," J. Display Technol., 12(6), 542-548 (2016).

[15] Navarro, H., Martínez-Cuenca, R., Saavedra, G., Martínez-Corral, M., and Javidi, B., "3D integral imaging display by smart pseudoscopic-to-orthoscopic conversion (SPOC)," Opt. Express, 18(25), 25573-25583 (2010).

[16] Martínez-Corral, M., Dorado, A., Navarro, H., Saavedra, G., and Javidi, B., "Three-dimensional display by smart pseudoscopic-to-orthoscopic conversion with tunable focus," Appl. Optics. 53(22), E19-E25 (2014).

[17] Shen, X., Xiao, X., Martinez-Corral, M., and Javidi, B., "Format matching using multiple-planes pseudoscopic-toorthoscopic conversion for 3D integral imaging display," Proc. SPIE 9495, Three-Dimensional Imaging, Visualization, and Display 2015, 94950W (2015). 